\title{
Secondary electron emission-capacitive probes for plasma potential measurements in plasmas with hot electrons
}

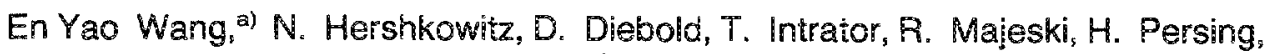 \\ G. Severn, B. Nelson, and Y. L. Wen' \\ University of Wisconsin-Madisor, Madison. Wisconsin 53706
}

(Received 23 October 1986; accepted for publication 2 February 1987)

\begin{abstract}
It is shown that a secondary electron emission-capacitive probe can determine the plasma potential when $T_{e} \geqslant 50 \mathrm{eV}$. The probe is wideband ( $1 \mathrm{~Hz}$ to greater than $20 \mathrm{MHz}$ ) and relativeiy simple to operate. The Phaedrus- 3 tandem mirror plasma where $T_{e} \sim 40-60 \mathrm{eV}$ and $n \simeq 5 \times 10^{12} \mathrm{~cm}^{-3}$ is used to verify this technique.
\end{abstract}

\section{ENTRODUCTION}

Plasma potential is an important parameter in "fusion" experiments because it affects radial transport in toroidal devices $^{1}$ and both radial and axial confinement in open systems. ${ }^{2}$ While swept Langmuir probes provide a convenient technique for determining plasma potential in many laboratory plasmas, ${ }^{3}$ the high-energy density of "fusion" plasmas have limited their use to plasma boundaries. In this region, fuctuations in density and potential are particularly high, further complicating their use. One technique for determining the potential and for observing the fuctuations has been to monitor the probe floating potential. However, the fioating potential is sensitive to the electron temperature and electron tails and may not be a good measure of the plasma potential. For example, consider a situation where the plasma potential is given by $3 T_{e} / e$, where $T_{e}$ is the plasma electron temperature. If the floating potential is $3.5 T_{e} / e$ more negative than the plasma potential, an increase in $T_{e}$ will result in an increase in the plasma potentiai but a decrease in the foating potential.

Another technique has been to follow the fioating potential of a heated emissive probe. ${ }^{4}$ This technique is limited by the ability of the probe to emit as much current as it collects, which in practice often requires that the probe be molten. Nevertheless, self-emissive probes which are heated by the plasma, have been shown to give a good measurement of the plasma potential. ${ }^{5}$ Unfortunately, such probes rarely last for more than one plasma discharge in pulsed fusion plasmas. Recently, it has been shown in laboratory plasmas 6 that secondary electron emission probes can replace thermionic emission probes. In those experiments, monoenergetic primary electrons produced the secondary emission. Secondary electron emission probes are particularly suited to "fusion" plasmas in which hot Maxwellian electrons produce the secondary electrons. Secondary electron emission increases with electron temperature and can be quite high. In this paper we report the first use of secondary electron emission probes in "fusion" plasmas for potential measurements. It is shown that it is relatively easy to construct and operate secondary electron-capacitive probes (SECP) which are smail in size, have good frequency response, and are much more durable than the hot thermionic emissive probes.

The energy distribution of the emitted secondary elec-

\footnotetext{
"On leave from Southwestern Institute of Physics, Leshan, Sichuan, China.
}

trons resulting from electron impact on solids has been found to be similar in character for all solids. ${ }^{7}$ All distributions measured for metals have their maxima near $2 \mathrm{eV}$, the exact shape depending on the metal and the nature of its surface. The total secondary electron current yield $\sigma$, defined as the ratio of the current of emitted electrons produced to the primary electron current, increases from a low value at small primary energies to a maximum value of $\sigma_{m}$ (usually greater than 1.0) at $E_{m}$, and then falls slowly with increasing primary energy. In general, the yield for metals and semiconductors is smaller than that for insulators. For instance, clear glass has a $\sigma \approx 2-3$ wher: primary energy exceeds $50 \mathrm{eV}{ }^{7}$ When primary electrons are Maxwellian, with temperature $T_{e}$, the effective secondary coefficient $\sigma_{\text {eff }}$ (Ref. 6) can also be greater than 1.0 for sufficiently high $T_{p}$.

A test of the SECP was carried out using Phaedrus-B, which is a five-cell tandem mirror. ${ }^{8} \mathrm{~A}$ central cell is bounded by thermal barrier cells which in turn are bounded by plug end celis. Plasma in Phaedrus-B is produced by ICRF (ion cyclotron range of frequencies) antennas at each end of the central cell and end cells. ${ }^{8}$ ICRF power is used for ion heating and $\mathrm{rf}$ stabilization. Representative plasma parameters are central cell ion density $n_{c}=5 \times 10^{12} \mathrm{~cm}^{-33}$, electron and ion temperatures, $T_{e} \approx 60 \sim 80 \mathrm{eV}$, and $T_{i} \approx 50 \mathrm{eV}$, barrier and end cell densities are $n_{b} \leqslant 0.2 n_{c}$ and $n_{e c} \sim n_{c} / 2$, respectively. Electron temperatures in the barrier and end cells are comparable to the central cell. For such electron temperatures, $\sigma_{\text {eff }} \simeq 1.0$ (Refs. 6 and 7 ) and the typical secondary electron energy $T_{s}$ always satisfies $T_{s} \ll T_{e}$. End cell ion temperatures are $\sim 500 \mathrm{eV}$. We found that plasma potential fluctuations in the central cell were approximately $100-300 \mathrm{~V}$ (peak-to-peak). These strong fiuctuations and the high-energy density make it difficult to use Langmuir and thermionic emission probes. A method of measurement of plasma potential fluctuations which makes use of time-averaged emitting probe $I-V$ characteristics has recently been presented. ${ }^{9}$ However, the technique only works with sinusoidal waves. The SECP technique can overcome these difficulties.

\section{SECONDARY ELECTRON EXISSION-CAPACITIVE PROBE}

\section{A. Floating potential of secondary emissive capacitive probe}

Probes can be considered to be one dimensional if the electron gyroradius is small compared to the probe radius. 
This condition is almost always satisfied in fusion experiments (except for self-emissive probes) and will be assumed throughout this paper. The possible potential structures between a foating probe and the ambient plasma are restricted by two requirements: that the net current to the probe is zero (the floating condition requirement) and that the plasma potential and density are related through Poisson's equation. Three qualitatively distinct potential structures for the sheath between an emitting probe and the plasma surrounding the probe have been suggested.,10-12 These structures are shown in Figs. 1 (a), 1(b), and $1(c)$.

A nonemitting probe's floating potential in a hydrogen plasma is approximately $3.5 T_{e} / e$ more negative than the plasma potential. ${ }^{3}$ With electron emission, a probe's foating potential becomes more positive. ${ }^{13}$ Space-charge effects can be neglected when electron emission is weak enough that the potential still increases monotonically between the probe and the ambient plasma. For such weak emission all the emitted electrons are able to escape from the probe to the plasma. With strong emission ( $\left.\sigma_{\text {eff }} \gtrsim 1\right)$, the floating condition requirement can only be satisfied if there is a potential dip near the probe which reduces the amount of emitted electron current that reaches the ambient plasma. For strong emission, the floating condition requirement can be satisned by all three of the potential structures corresponding to Fig. 1 by suitable choice of the potential dip.

Chen has argued that, ${ }^{3,12}$ when the typical energy of the secondary electrons $T_{s}$ is much less that $T_{e}$, the value of the floating potential is not brought much closer to the plasma potential by emission. This situation is shown in Fig. $1(a)$. Most of the emitted current is reflected back by a potential $\operatorname{dip} \Delta \phi_{i}$ which satisfies $T_{e}>3 \Delta \phi_{1}>T_{s}$. For such a potential structure, the emitted electrons can be ignored in considering net current to the probe and the floating potential of the strongly emitting probe is the floating potential of a non. emitting object within a few $T_{s} / e$.

The potential structure corresponding to Fig. $\Omega(a)$ is not consistent with Poisson's equation. Since little of the emitted current in Fig. 1(a) reaches the minimum of the potential dip point $M$ and because the potential at point $M$ is nearly the floating potential of a nonemitting probe, the density of electrons and ions at point $M$ is nearly the same as that

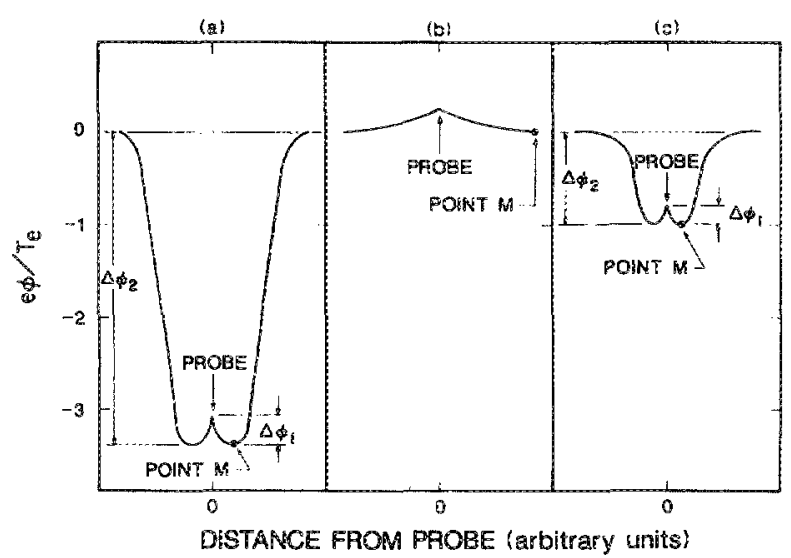

FIG. 1. Three-probe sheath potential structures which satisfy the floating condition requirement. at a nonemitting probe. Hence, at point $M$ the density ratio of electrons to ions is

$$
\left(n_{\mathrm{e}} / n_{i}\right) \approx \sqrt{2 e\left[\left(V_{p}-V_{f}\right) / T_{e}\right]\left(m_{e} / m_{i}\right)} \leqslant 1,
$$

where $V_{f}$ is the floating potential of a nonemitting probe and $V_{p}$ is the plasma potential. This is inconsistent with the Poisson's equation requirement that $\left(n_{e} / n_{i}\right)>1$ at a point such as point $M$, where the second derivative of potential is positive. The only way to simultaneously increase $n_{e} / n_{i}$, and to satisfy the fioating condition is to reduce $\Delta \phi_{2}$.

Sizonenko ${ }^{10}$ has argued that when strong secondary electron emission is present, the floating potential has two possible values, one above the ambient plasma potential Isee Fig. 1(b)] and one approximately $T_{e} / e$ below [see Fig. 1(c)]. He further argues that the solution the plasma finds depends on the piasma initial conditions. The validity of the solution Fig. I (b), above the ambient plasma potential, is questionable because Sizonenko assumes that the ratio of the ambient ion dersity to the ambient electron density is a free parameter which can adjust to the extra electron density to the emitted from the probe, even if the emitted electron density is a large perturbation to the ambient plasma.

The potential structure corresponding to Fig. 1(b) satisfies the fioating condition requirement, but not Poisson's equation, if the ratio of the ambient ion density to the ambient electron density is not treated as a free parameter. The current of emitted electrons, which reaches point $M$, must approximately equal the current of plasma electrons flowing towards the probe in order to satisfy the floating condition requirement. Since the currents are nearly equal, the ratio of emitted electron density to plasma electron density at point $M$ in $F i g, 1(b)$ is approximately $\left(T_{e} / T_{s}\right)^{1 / 2}$, which is greater than one (a typical value is 3 ). Quasineutrality at point $M$ requires that $n_{e} \approx n_{i}$ which can only be achieved if the ratio of the ambient ion density to ambient electron density is dramatically increased.

Sizonenko ${ }^{10}$ and others ${ }^{11}$ have argued that when the secondary emission emission $\sigma_{\text {eff }}$ is greater than one, the potential structure between the probe and the plasma can be that corresponding to Fig. 1(c). This potential structure satisfies both the floating condition requirement and Poisson's equa. tion and resernbles Fig. 1(a) except that potential dip with respect to the ambient plasma $\Delta \phi_{2}$ in Fig. $1(\mathrm{c})$ is about $\frac{1}{3}$ the value of $\Delta \phi_{2}$ in Fig. I (a). The ratio of the emitted electron density to plasma electron density at point $M$ in Fig. $1(c)$ is about $\left(T_{e} / T_{s}\right)^{1 / 2}$ when the floating condition requirement is met. For approximately constant ion density in the sheath, $\Delta \phi_{2} \leqslant T_{e} / e$ gives a net electron density at point $M$, which is required for consistency between the potential structure and Poisson's equation. The analytical solution to $\Delta \phi_{2}$ of Fig. $l(c)$ as found by Sizonenko ${ }^{10}$ is

$$
\begin{aligned}
(2 / \alpha) & {\left[\left(1+\alpha \psi_{m}\right)^{1 / 2}-1\right] } \\
= & \left\{1+\Phi\left[\left(\psi_{m}\right)^{1 / 2}\right]+\left[e^{-\psi_{m}}\left(\pi \psi_{m}\right)^{1 / 2}\right]\right\}^{-1} \\
& \times\left\{1+\Phi\left[\left(\psi_{m}\right)^{1 / 2}\right]-e^{-\psi_{m}}\right\},
\end{aligned}
$$

where

$$
\Phi\left[\left(\psi_{m}\right)^{1 / 2}\right] \equiv 2(\pi)^{-1 / 2} \int_{0}^{\psi_{m}^{1 / 2}} e^{-i^{2}} d t
$$




$$
\begin{aligned}
& \psi_{m} \equiv e \phi_{m} / T_{e}, \quad \phi_{m}=\Delta \phi_{2}, \\
& \alpha=\left(2 T_{e} / m_{i} u^{2}\right) .
\end{aligned}
$$

Far away from the probe, Sizonenk ${ }^{10}$ assumes that the ions are monoenergetic and heading towards the wall with velocity $u$.

Figure 2 gives $e \Delta \phi_{2} / T_{e}$ vs $\left(m_{i} u^{2} / 2 T_{e}\right)$ corresponding to Eq. (2), and it shows $0.5 \leqslant\left(e \Delta \phi_{2} / T_{e}\right) \leqslant 0.9$. The upper limit is determined by the Bohm Sheath Criteria that the minimum value of $m_{i} u^{2} / 2 T_{e}$ is 0.5 for $T_{i} \leqslant T_{e}$. For increasing values of $\left(m_{i} u^{2} / 2 T_{e}\right)$, the solution approaches 0.5 . Note that Eq. (4) predicts that $\Delta \phi_{2}$ is only weakly dependent on $u$ and roughly equal to $T_{e} / e$. In the derivation ${ }^{10}$ of Eq. (2), it was assumed that the ratio of the ambient ion density to the ambient electron density is able to adjust to the emitted electrons in order to preserve quasineutrality. In Fig. I(c), the emitted electron density in the plasma is only $0.2-0.4$ the ambient electron density. The ambient electron density can readily adjust to these emitted electrons. Therefore, it appears that of the three choices in Fig. 1, only the potential structure shown in Fig. I(c) is consistent with Poisson's equation and the ffoating condition.

Strong magnetic fields accentuate the perturbation of a probe on the plasma when the currents drawn to and from the probe are confined to the flux tube containing the probe and its sheath. As compared to a current collecting Langmuir probe, a nonemitting fioating probe is a small perturbation because it draws no net electrical current and very little electron current from the plasma. Hence, there is no charging and relatively little electron depletion of a fux tube containing a nonemitting foating probe. An emitting floating probe corresponding to Fig. I (c) has the advantage of being much closer to the plasma potential than a nonemitting probe while still drawing no net current. Furthermore, the net particle current between a plasma and a floating probe is constant regardless of the amount of probe emission. As the emission (and hence the potential) of a floating probe is increased, the plasma electron current drawn by the probe and the emitted electron current to the plasma both increase by the same amount to satisfy the foating condition requirement. Hence, as emission is increased, there is no net increase in particle loss to the probe. Note, though, that powe: loss to the probe increases as emission increases, the more energetic plasma electrons being replaced by the less ener. getic electrons.

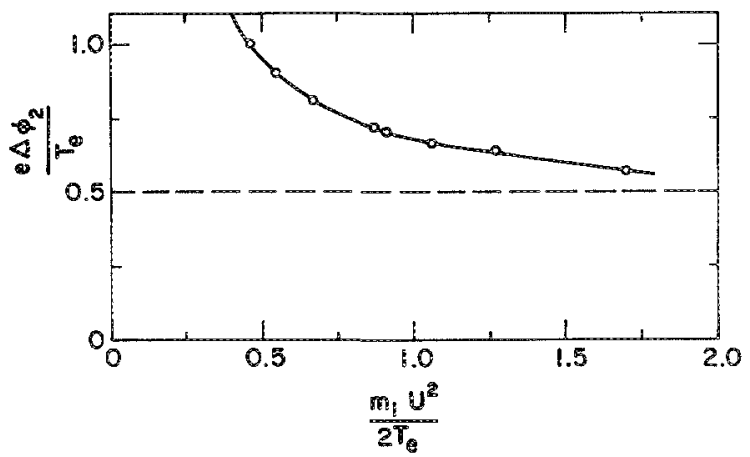

FIG. 2. The probe sheath potential dip vs the energy of ions entering the sheath for potential structures corresponding to Fig. 1 (c) and Eq. (2).

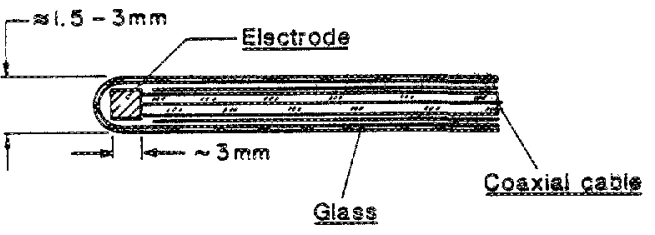

FIG. 3. The physical construction of a capacitive probe.

\section{B. Probe construction and circuit}

The capacitive probes used consisted of a glass (or ceramic) envelope and a detecting electrode. The prisical construction of a typical probe appears in Fig. 3. The input capacitance was determined by the dimensions and the glass, and the dielectric properties of the glass. To obtain a satisfactory frequency response, the load was chosen to be largely capacitive. A resistor $R$ was placed in parallel with a high input impedance operational amplifier to prevent the input bias current of the amplifier from charging the input capacitance to large voltages. The equivalent circuit at low frequency is shown in Fig. 4. The capacitance $C_{1}$ as the coupling between the plasma and the electrode while $C_{2}$ was the capacitance of the cable and amplitude. The low-frequency cutoff $f_{L}$ was $1 /\left(2 \pi R_{i} C_{2}\right)$, where $R_{i}$ is the effective resistance of resistor $R$ in parallel with the input resistance of the amplifier. The probe attenuation was equal to $C_{1} /\left(C_{1}+C_{2}\right)$. Typical parameters were: $C_{1} \approx 0.3-0.6 \mathrm{pF}$, $C_{2} \approx 0.01 \approx 0.03 \mu \mathrm{F} ; R=88 \mathrm{M} \Omega, R_{i} \cong R, f_{L} \approx 1 \mathrm{~Hz}, C_{1} /$ $\left(C_{1}+C_{2}\right) \approx C_{1} / C_{2} \approx 1 \times 10^{-5}$ to $6 \times 10^{-5}$. The duration of discharge in Phaedrus-B is $\sim 20 \mathrm{~ms}$ so this circuit is adequate for measuring "dc" levels of plasma potential. This system can extend the high-frequency response up to $\sim 100$ $\mathrm{MHz}^{14}$

\section{Callbration procedure and typical}

Calibration was performed by covering the probe with aluminum foil or inserting the probe into mercury as shown in Fig. 5(a). The low-frequency response of the probe was measured by applying a $20-\mathrm{ms}$ duration square signal $V_{\text {in }}$ to the aluminum foil. Both $V_{\text {in }}$ and the correspondisg output signal $V_{\text {out }}$ from the follower are shown in Fig. 5(b). The attenuation $V_{\text {out }} / V_{\text {in }}$ was approximately $10^{-4}$. The high-frequency response of the probe was measured up to $20 \mathrm{MHz}$ where the attenuation was found to be less than $3 \mathrm{~dB}$. The probe frequency response satisfies the requirements of Phaedrus-B experiments.

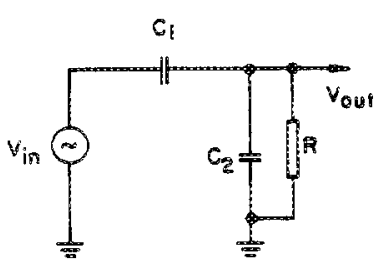

FIG. 4. The low-frequency equivalent circuit for the capacitive probe. 


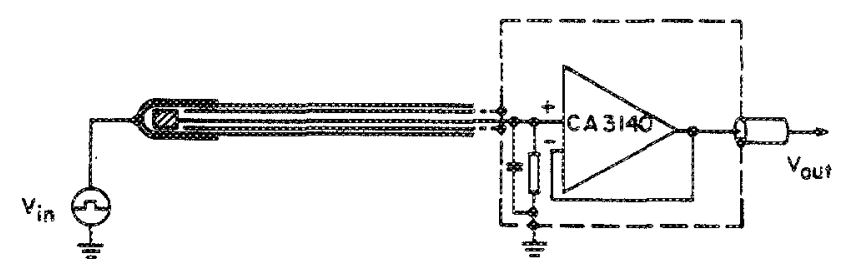

c)

b)

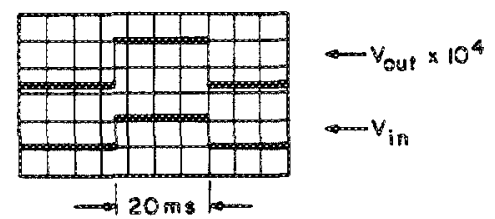

FIG. 5. (a) Calibration test circuit for capacitive probe. (b) Waveform of $V_{\text {in }}$ and $W_{\text {out }}$ showing a low-frequency response.

\section{U1. SECP TECHNIQUE FOR MEASURING PLASMA POTENTIAL.}

The theory suggests that the floating potential of a SECP is $(0.7 \pm 0.2)\left(T_{e} / e\right)$ lower than the plasma potential. Hence, if $T_{e}$ is known, the plasma potential can be calculated from the SECP floating potential.

Unfortunately, in the Phaedrus-B tandem mirror the electron temperature is not well known. For a nonemitting probe, $\phi_{p}-\phi_{f} \simeq 3.5 T_{e} / e$, where $\phi_{p}$ is the plasma potential and $\phi_{f}$ is the floating potential of the probe. This implies

$$
\phi_{s}-\phi_{L}=(2.8 \pm 0.2) T_{e} / e
$$

and

$$
\phi_{p} \simeq \phi_{s}+0.7\left[\left(\phi_{s}-\phi_{L}\right) / 2.8\right],
$$

where $\phi_{s}$ and $\phi_{L}$ are the floating potential of a SECP and a Langmuir probe, respectively. Data in Fig. 6 show that the SECP floating potential is much more positive than the floating potential of a metal Langmuir probe. The foating potential of a clean Langmuir probe, with little secondary emission, was $\simeq-175 \mathrm{~V}$ [see Fig. 6(a)], while the floating potential of a capicitance probe with a strong secondary emission was $\simeq+75 \mathrm{~V}$ [see Fig. $6(\mathrm{~b})$ ]. The electron termperature $T_{s}$, measured by a double probe at the floating potential, was $\sim 80 \mathrm{eV}$.
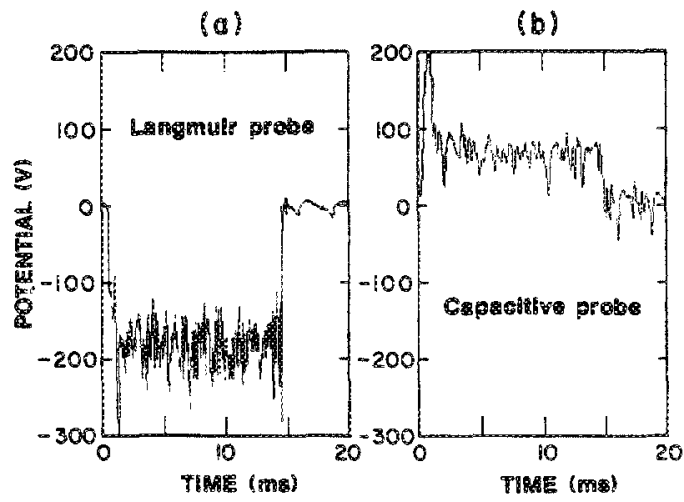

FIG. 6. (a) Langmuir probe foating potential and (b) SECP floating potential vs time.

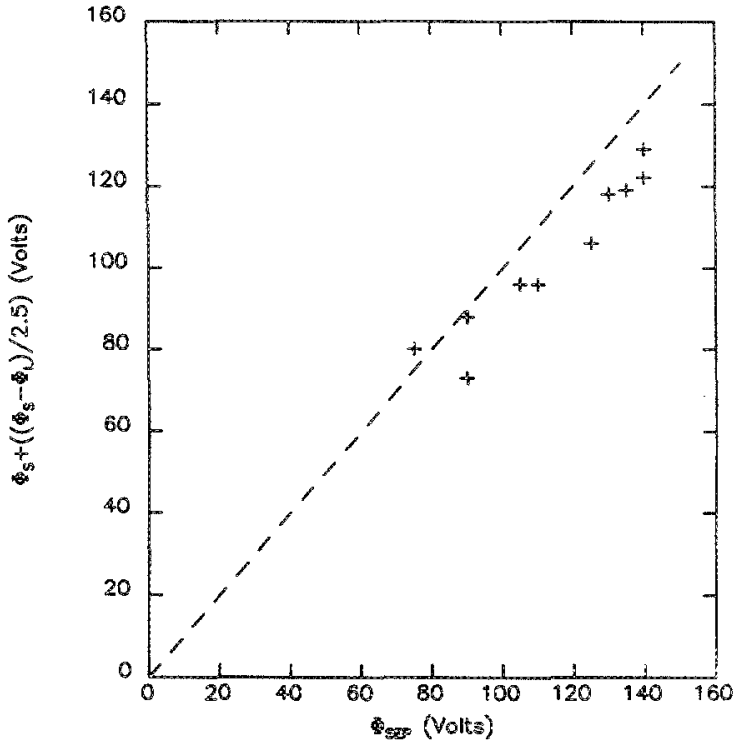

FIG. 7. Data graph as $\phi_{s}+\left[\left(\phi_{s}-\phi_{L}\right) / 2.5\right]$ vs $\phi_{\mathrm{SEP}}$, where $\phi_{s} \phi_{L}$, and $\phi_{\text {SEP }}$ are the foating potentials of the SECP, Langmuir probe, and self-emissive probe, respectively.

Figure 7 is Phaedrus $-\mathbb{B}$ data graphed as $\phi_{s}+\left[\left(\phi_{s}-\phi_{L}\right) / 2.5\right]$ vS $\phi_{\mathrm{SEP}}$, where $\phi_{\mathrm{SEP}}$ is the floating potential of a self-emissive probe. As noted before, ${ }^{4}$ $\phi_{\text {SEP }} \simeq \phi_{p}$. Figure 7 shows empirically that

$$
\phi_{p} \approx \phi_{s}+\left[\left(\phi_{s}-\phi_{X}\right) / 2.5\right] \text {. }
$$

It should be noted that Fig. 7 contains only data for which

$$
\left[\left(\phi_{s}-\phi_{L}\right) / 2.5\right]>30 \mathrm{eV} .
$$

If the electron temperature is too low, $\sigma_{\mathrm{eff}}<1$ and the SECP is not a good measure of plasma potential, Eq. (5) is not valid. In agreement with theory, it was found that data for which Eq. (6) was not valid fell much below the dashed line in Fig. 7. Hence, Eq. (6) is a good test for the sufficiently high electron temperature which is necessary for the accuracy of the SECP method, Eq. (5).

Equation (5) is in slight disagreement with Eq. (4), but this disagreement can be accounted for. Electron temperature calculated from Eq. (3) will tend to be lower than the actual electron temperature because of secondary emission (a)

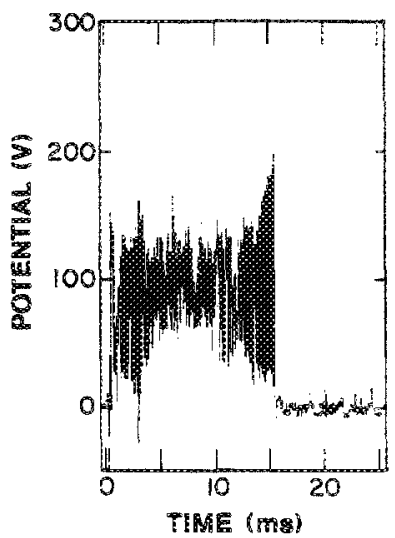

(b)

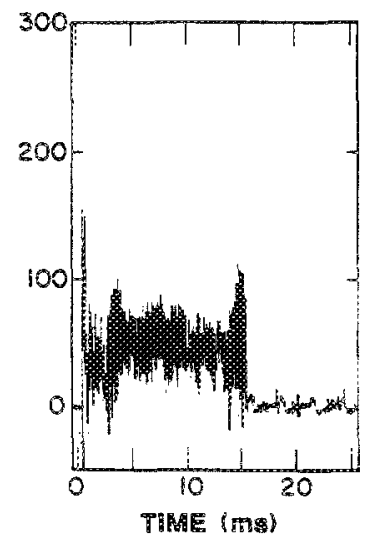

FIG. 8. Effects of surface conditions on fiosting potential: (a) data for clean surface, (b) data after titanium was deposited on the probe surface. 
from the Langmuir probe. An effective secondary coefficient of 0.7 for the Langmuir probe would account for the discrepancy between Eqs. (4) and (5). In Phaedrus, titanium gettering is used for vacuum pumping. Titanium has a secondary emission coeficient $\sigma_{\text {eff }} \approx 0.7$ for $T_{e} \simeq 40 \mathrm{eV}^{15}$ a roughly typical electron temperature for Phaedrus-B. No effort was made to keep the surface of the Langmuir probe clean of titanium. Hence, the discrepancy between Eqs. (4) and (5) can be accounted for by a titanium coating of the Langmuir probe. When the Langmuir probe surface is kept clean, and the probe is made of a weakly secondary emitting material such as tantalum, Eq. (4) is expected to be valid.

Figure 8 shows the effects that a thin fim of titanium on the surface of the SECP has on the measured foating potential of the SECP. Figure $8(a)$ gives data for a clean SECP while Fig. $8(b)$ gives data at the same point in the plasma after a thin film of titanium was deposited on the SECP. A significant decrease of the floating potential of the SECP is apparent after the titanium was deposited, as is expected because glass is a stronger secondary emitter than titanium. The SECP was pulled out of the machine between shots in order to keep the SECP free of titanium.

\section{ACKNOWLEDGMENTS}

We thank Dr. R. A. Breun for helpful suggestions. This work was supported by DOE Contract DE-ACO2. 78 ET51015 and NSF Grant ECS-8313388.
'R. J. Taylor, B. W. James, S. X. Jin, L. Keller, P. S. C. Lee, N. C. Luhmann Ir., G. J. Morales, L. Oren, H. Park, W. A. Peebles, S. Talmadge, and C. X. Yu, in Plasma Physics and Controlled Nuclear Fusion Research, 1982 (Proceedings 9th International Conference, Baltimore, 1982) (IAEA, Vienna, 1983), Vol. IEI, p. 251.

${ }^{2} \mathrm{E}$. B. Hooper, R. H. Cohen, D. L. Correll, J. M. Gilmore, and D. P. Grubb, Phys. Fluids 28, 3609 (1985).

${ }^{3}$ F. F. Chor, Plasma Diagnostic Techniques, edited by Huddlestone and Leonard (Academic, New York, 1965), pp. 184 and 185.

${ }^{4}$ N. Kershkowitz, J. Pew, Nelson, and D. Gates, Rev. Sci. Instrum. 54, 29 (1983).

${ }^{5}$ N. Hershkowitz, B. A. Nelson, J. Johnson, J. R. Ferron, H. Persing, C. Chan, S. N. Golovato, J. D. Callen, and J. Woo, Phys. Rev. Lett. 55, 947 (1985).

${ }^{6} \bar{E}$. Y. Wang, N. Herskkowitz, and T. Intrator, Rev. Sci. Instrum. 57, 1085 (1986).

D. J. Gibbons, in Handbook of Vacuum Physics, edited by A. H. Beck (Pergamon, New York, 1966), Vol. 2.

${ }^{8}$ R. A. Breun, P. Brooker, J. Browning, D. Brouchous, G. Butz, J. Conrad, E. Dales, J. Ferron, R. Goulding, N. Hershkowitz, T. Intrator, C. Litwin, R. Majeski, S. Meassick, B. Nelson, L. Peranich, $\mathbb{H}$. Persing, J. Radtke, D. Roberts, G. Severn, D. Sing, and En Yao Wang, in Proceedings of the 17th International Conference on Plasma Physics and Controlled Nuclear Fusion Research, Kyoto, 1986 (International Atomic Energy Agency, Vienna) (IAEA, Vienna, 1986), Paper IAEA-CN-47/C-I-4-1.

${ }^{9}$ E. Y. Wang, N. Hershkowitz, T. Intrator, Kev. Sci. Instrum. 57, 2425 (1986).

1"V. L. Sizonenko, Sov. Phys. Tech. Phys. 26, 1345 (1981) [Zh. Tekh. Fiz. 51, 2283 (Noy. 1981)].

"G. D. Hobbs and T. A. Wesson, Plasma Phys. 9, 85 (1967); P. T. Harbour, Preprint CLM-P535, Culham (1978).

${ }^{12}$ F. F. Chen, Nagoya University, Institute of Plasma Physics, Report No. IPPJ-750, Nagoya, Japan (1985).

${ }^{13}$ R. F. Kemp and J. M. Sellen, Jr., Rev. Sci. Instrum, 37, 455 (1966).

${ }^{14}$ J. A. Schmidt, Rev. Sci. Instrum. 39, 1297 (1968).

15O. Hachenberg and W. Braurer, Eiectronics and Electron Physics $X I$ (Academic, New York, 1959). 